\title{
Correction to: Fis1 depletion in osteoarthritis impairs chondrocyte survival and peroxisomal and lysosomal function
}

\author{
Dongkyun Kim ${ }^{1}$ - Jinsoo Song ${ }^{1} \cdot$ Yeonho Kang ${ }^{1}$ - Sujung Park ${ }^{1}$ - Yong-II Kim ${ }^{2}$ - Seongae Kwak ${ }^{2}$ - Dongkwon Lim ${ }^{3}$. \\ Raekil Park ${ }^{2}$. Churl-Hong Chun ${ }^{4} \cdot$ Seong-Kyu Choe ${ }^{2,5} \cdot$ Eun-Jung Jin ${ }^{1,5}$
}

Published online: 19 November 2019

(C) Springer-Verlag GmbH Germany, part of Springer Nature 2019

Correction to: J Mol Med (2016) 94:1373-1384

https://doi.org/10.1007/s00109-016-1445-9

In Fig. 1C, the wrong si-control image was used by mistake. In the corrected version of Fig. 1C, the correct image of the sicontrol is shown. The authors claim that this correction does not affect the conclusion of the study and apologize for the oversight.

The online version of the original article can be found at https://doi.org/ 10.1007/s00109-016-1445-9

Seong-Kyu Choe

seongkyu642@wu.ac.kr

$\triangle$ Eun-Jung Jin

jineunjung@wku.ac.kr

1 Department of Biological Sciences, College of Natural Sciences, Wonkwang University, Iksan, Chunbuk 570-749, South Korea

2 Department of Microbiology, Wonkwang University School of Medicine, Iksan, Chunbuk 570-749, South Korea

3 KU-KIST Graduate School of Converging Science and Technology, Korea University, Seoul 136-701, South Korea

4 Department of Orthopedic Surgery, Wonkwang University School of Medicine, Iksan, Chunbuk 570-749, South Korea

5 Integrated Omics Institute, Wonkwang University, Iksan, Chunbuk 570-749, South Korea
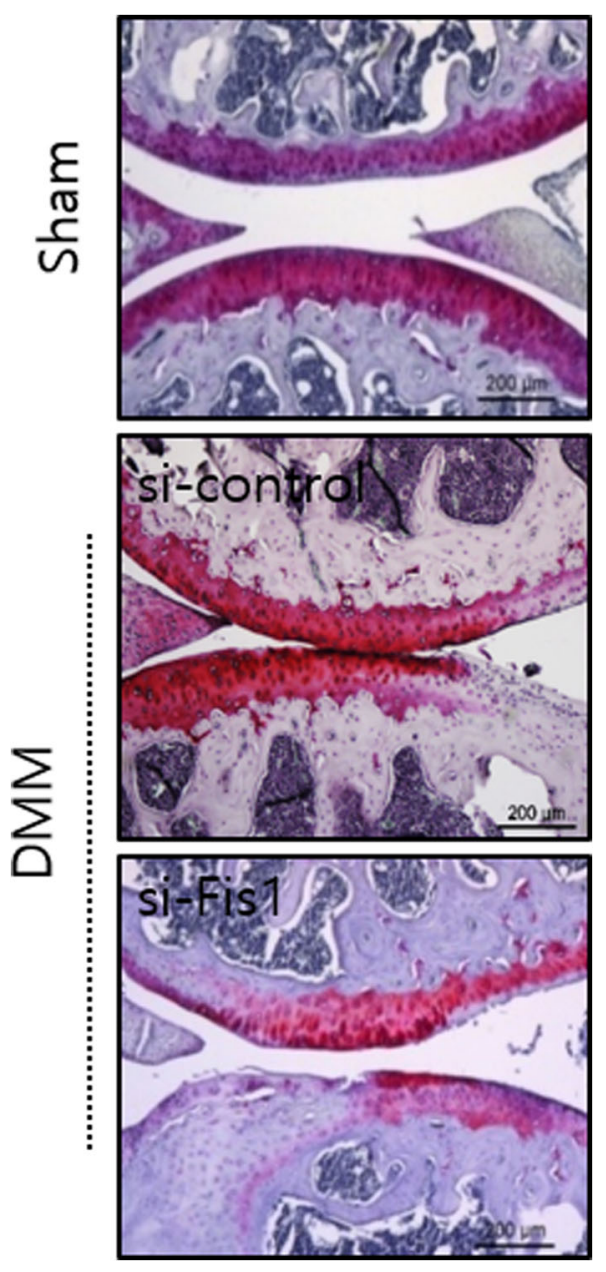